\title{
Electron cloud buildup and instability: Numerical simulations for the CERN Proton Synchrotron
}

\author{
M. Giovannozzi, E. Métral, G. Métral, G. Rumolo, and F. Zimmermann \\ CERN, CH 1211 Geneva 23, Switzerland
}

(Received 3 June 2002; revised manuscript received 31 October 2002; published 6 January 2003)

\begin{abstract}
Experimental observations on the electron cloud have been collected at the CERN Proton Synchrotron machine throughout the last two years. At the same time, an intense campaign of simulations has been carried out to understand the observed electron cloud buildup and the related instability. In this paper, the results of the numerical simulations are presented and discussed.
\end{abstract}

DOI: 10.1103/PhysRevSTAB.6.010101

\section{INTRODUCTION}

Gas ionization and electron multiplication due to the secondary-emission process on the inner side of the beam pipe may induce the buildup of an electron cloud, which can significantly degrade the performance of rings operating with closely spaced proton or positron bunches. The undesired electron cloud causes pressure rise and beam instability when the parameters are pushed above certain thresholds [1].

In the case of the CERN Proton Synchrotron (PS) machine, the electron cloud has been observed since the year 2000 for LHC-type bunch trains, i.e., 72 bunches of $N_{b}=11 \times 10^{10}$ protons per bunch $(\mathrm{p} / \mathrm{b})$ spaced by $25 \mathrm{~ns}$. The baseline drift in the beam-position monitor signal caused by electrons impacting on the electrostatic pickup electrodes during the passage of a bunch train, as well as observations with secondary-emission grid profile monitors, has given evidence of the presence of a large number of electrons inside the beam chamber [2]. To better quantify the degrading effects of the electron cloud on the machine performance, measurements were carried out with an LHC beam stored in the PS at high momentum $(26 \mathrm{GeV} / c)$ for an extended period of time (Ref. [3] describes further details of the applied beam manipulations as well as the PS beam observations with an electron cloud). Under these conditions, data could be recorded concerning not only the buildup process, but also the induced instability [3]. The main experimental observations on the electron cloud driven instabilities in the PS can be summarized as follows. The instability manifests itself as a single-bunch phenomenon which sets in above an intensity threshold of about $4-5 \times 10^{10} \mathrm{p} / \mathrm{b}$ and is especially evident in the horizontal plane. Its rise time is about 3-4 $\mathrm{ms}$ and it causes a transverse emittance growth which can be as large as a factor 10 or 20 in the horizontal plane and 2 in the verticzal one.

The two codes developed at CERN, ECLOUD and HEADTAIL [4], can simulate both the buildup process of the electron cloud and its expected effect on the single bunch that passes through it [1,5-7]. This paper reports on the results of numerical simulations carried out with these two codes.
PACS numbers: 29.27.Bd, 29.27.Fh, 29.20.Lq, 07.05.Tp

Section II gives a short description of the PS lattice and its main magnets. Section III is devoted to results of buildup simulations for the combined function magnet regions of the CERN PS, considering different bunch intensities and lengths. Using the saturation value and distribution of the cloud density as obtained in Sec. III, a full instability study in combined function magnets via computer simulations is presented in Sec. IV. Emphasis is placed on the expected dependence of the instability evolution on key parameters such as the bunch intensity, chromaticity, and bunch length. Simulation results are compared with observations in Sec. V. Conclusions are drawn in Sec. VI.

\section{PS LATTICE AND MAIN MAGNETS}

The PS lattice consists of ten superperiods each made of ten combined function magnets $4.26 \mathrm{~m}$ long, interlaced with eight $1.0 \mathrm{~m}$ and two $2.4 \mathrm{~m}$ drift spaces [8]. Every magnet is composed of two half-units with gradients of opposite sign, separated by a central junction. Each halfunit is made of five blocks with small gaps in between. Additional field adjustment can be made using the three currents of the pole-face winding and figure-of-eightloop devices located on the magnet poles. These additional current loops allow controlling the machine tunes and chromaticities. The layout of a PS magnet unit in the extraction region is shown in Fig. 1.

The latest PS magnetic field measurements using Hall probes were undertaken in 1992 [9] for different operational settings of the currents in the main coil, pole-face and figure-of-eight-loop windings. The measurements have been carried out in the median plane of the laboratory test PS magnet unit U17 composed of two half-units. The data of the vertical field component, including measurements of the central field, the end and lateral stray fields, and the field in the junction between the two halfunits, produced a discrete 2D field map [9].

The field measurements were carried out in a Cartesian coordinate frame. The longitudinal $z$ axis coincides with the magnet axis and its orientation is given by the direction of motion of the protons (see Fig. 1). The radial $x$ axis 


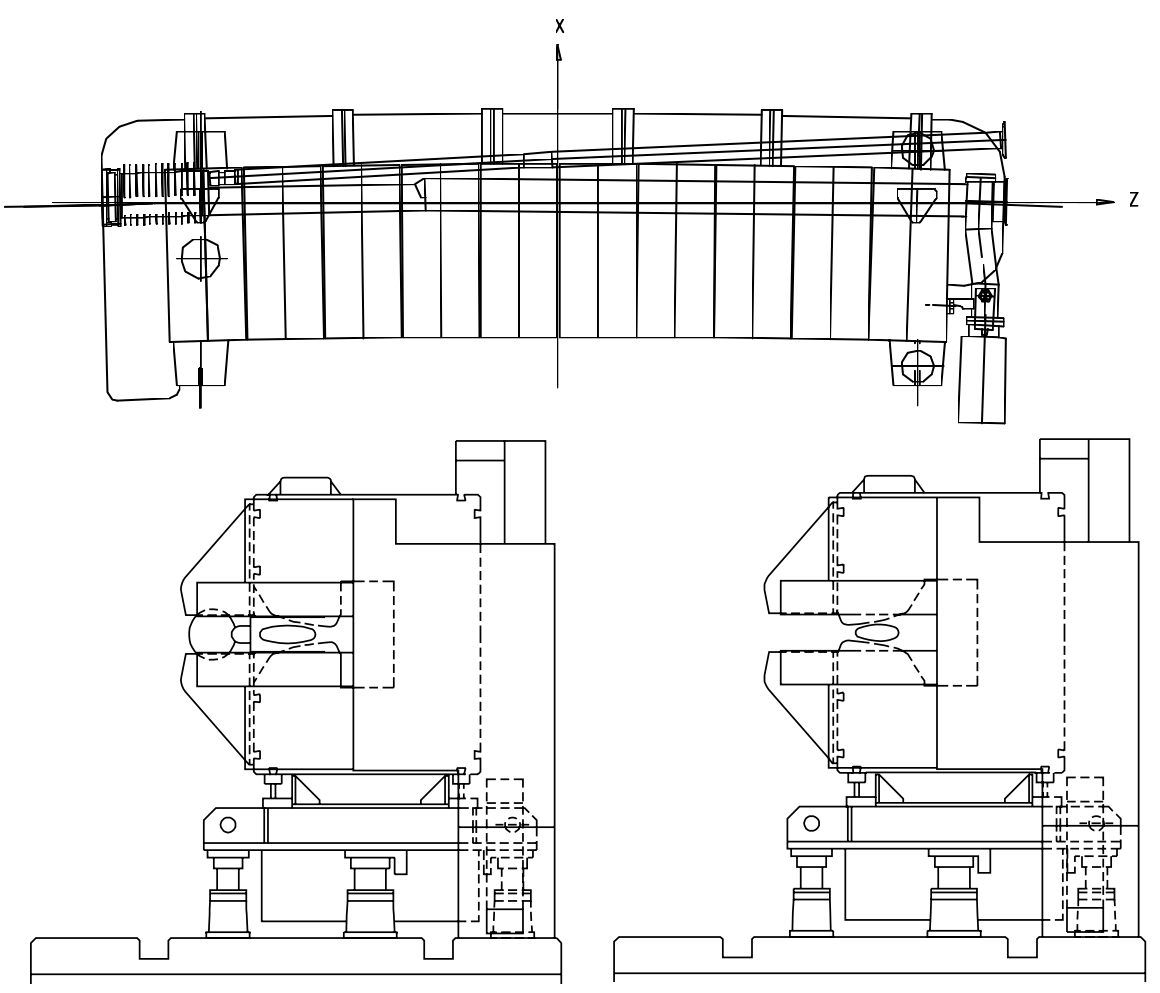

FIG. 1. PS magnet unit 16. This unit is located just downstream of the extraction septum. The overall layout is shown in the upper part. The vacuum pipes for the circulating beam as well as that for the extracted one are visible. The two cross sections of the entry face (with open gap) and exit face (with closed gap) of the magnet are also shown on the left and right, respectively.

coincides with the mechanical symmetry axis and it points towards the exterior of the PS ring (see Fig. 1). In this reference system a regular mesh is defined and for each point in the mesh, the value of $B_{y}$ has been measured in the median plane. The step size is $20 \mathrm{~mm}$ along the longitudinal $z$ axis and $10 \mathrm{~mm}$ along the radial $x$ axis. The mesh extends from -2.55 to $2.73 \mathrm{~m}$ and from -70 to $310 \mathrm{~mm}$ in the longitudinal and radial directions, respectively. The fitted 2D field map for the $26 \mathrm{GeV} / c$ working

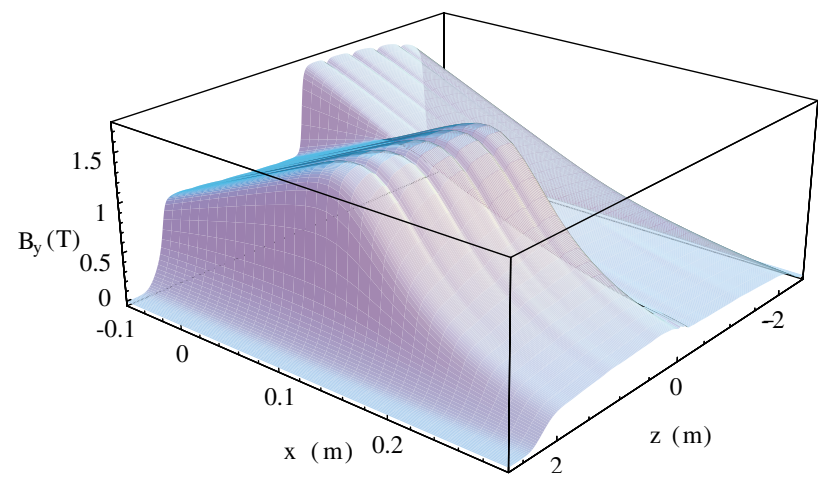

FIG. 2. (Color) Polynomial field map obtained by fit of the measured field values $B_{y}=B_{y}(x, z, 0)$ for the $26 \mathrm{GeV} / c$ working point. Here $x$ stands for the transverse coordinate, while $z$ is the longitudinal one. point is shown in Fig. 2 (see Ref. [10] for more details). In first approximation, this field can be modeled as

$$
B_{x}= \pm G y, \quad B_{y}=B_{y 0} \mp G x,
$$

with values for $B_{y 0}$ and $G$ given in Table I.

\section{ELECTRON CLOUD BUILDUP}

The simulation algorithm used in the ECLOUD code has already been discussed in great detail in previous papers (for instance, Ref. [5]). By simulating residual gas ionization and secondary emission at the chamber walls, including elastic reflection of low-energetic electrons, the code can calculate whether a high-density electron cloud is expected to form during the passage of a closely spaced bunch train. To simulate the passage of an LHCtype proton beam through a PS combined function magnet chamber, we assumed the parameters of Table I. The value of the secondary-emission yield for perpendicular incidence, $\delta_{\max }$, refers to stainless steel in the "asreceived" state [11], and $\epsilon_{\max }$ is the electron energy at which the electron yield reaches the maximum. An elastically backscattere component of secondary electrons is included according to a parametrization inferred from laboratory measurements [12]. A few primary electrons are needed in order to launch the multipacting cascade, 
TABLE I. PS parameters used in the simulations. As far as the beam emittance is concerned, the rms physical value is quoted here.

\begin{tabular}{lc}
\hline \hline Number of bunches $\left(k_{b}\right)$ & 72 \\
Bunch spacing $\left(T_{\text {sep }}\right)$ & $25 \mathrm{~ns}$ \\
Bunch population $\left(N_{b}\right)$ & $3-10 \times 10^{10} \mathrm{p} / \mathrm{b}$ \\
Transverse rms emittances $\left(\epsilon_{x, y}\right)$ & $0.09 / 0.09 \mu \mathrm{m}$ \\
Chamber half-apertures $\left(h_{x, y}\right)$ & $70 / 35 \mathrm{~mm}$ \\
Tunes $\left(Q_{x, y, s}\right)$ & $6.25 / 6.25 / 0.0015$ \\
Bunch rms length $\left(\sigma_{z} / c\right)$ & $1-4 \mathrm{~ns}$ \\
rms relative momentum spread $(\Delta p / p)$ & $1.75-7 \times 10^{-4}$ \\
Longitudinal $2 \sigma$ emittance $\left(\epsilon_{l}\right)$ & $0.35 \mathrm{eVs}$ \\
Circumference $(C)$ & $628 \mathrm{~m}$ \\
Dipole field $\left(B_{y 0}\right)$ & $1.256 \mathrm{~T}$ \\
Field gradient $(G)$ & $5.2 \mathrm{~T} / \mathrm{m}$ \\
Relativistic mass factor $(\gamma)$ & 27.7 \\
Revolution period $\left(T_{\text {rev }}\right)$ & $2.1 \mu \mathrm{s}$ \\
Average beta functions $\left(\beta_{x, y}\right)$ & $16 / 16 \mathrm{~m}$ \\
Average dispersion function $\left(D_{x}\right)$ & $2.56 \mathrm{~m}$ \\
Momentum compaction $(\alpha)$ & 0.027 \\
Chromaticities $\left(\xi_{x, y}=Q_{x, y}^{\prime} / Q_{x, y}\right)$ & up to $0.5 \mathrm{in}$ \\
& both planes \\
Maximum secondary emission yield $\left(\delta_{\max }\right)$ & 1.9 \\
Electron energy for maximum & \\
secondary-emission yield $\left(\epsilon_{\max }\right)$ & $300 \mathrm{eV}$ \\
Vacuum pressure $(\mathrm{CO})$ & $10 \mathrm{nTorr}$ \\
\hline \hline
\end{tabular}

which proceeds via electron acceleration in the beam field, electron loss to the wall between successive bunch passages, and subsequent secondary emission. In the simulation we consider a rate of primary "seed" electrons that corresponds to ionization of carbon-monoxide molecules at a pressure of $10 \mathrm{nTorr}$, assuming an ionization cross section of $2 \mathrm{Mb}$. We distribute the primary electrons uniformly in azimuthal angle around the surface of the chamber. In reality, the gas ionization should occur exclusively inside the beam volume. However, other processes such as beam loss or residual activation can generate primary electrons near the wall. We have verified that the average saturation density and the buildup time of the electrons do not depend on this difference in launch position [13].

Figure 3 depicts the simulated evolution of the electron line density $\lambda_{e}$ in a PS combined function magnet for the $1.8 \mu \mathrm{s}$ long bunch train. Various bunch lengths are considered, representing different snapshots during bunch compression prior to beam extraction (the bunches in the PS are compressed by a factor 4 , from 4 to $1 \mathrm{~ns}$ within about 100 turns). The simulation demonstrates that the electron line density grows faster the shorter the bunch, and that for the initial (uncompressed) bunch length of $\sigma_{z} / c=4 \mathrm{~ns}$ the electron cloud buildup appears only at the end of the bunch train.

Although the saturation starts earlier the shorter the bunch, the density at the moment of saturation is higher

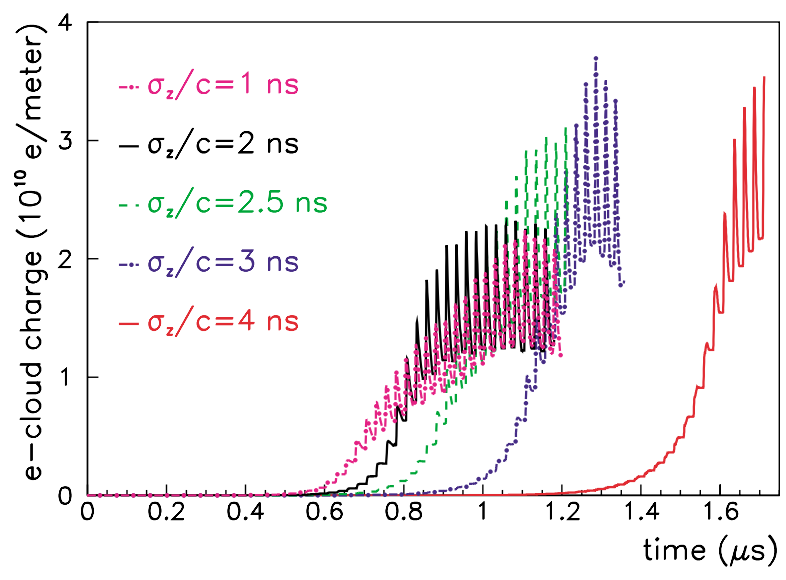

FIG. 3. (Color) Electron cloud buildup in a PS combined function magnet during the first $1.7 \mu \mathrm{s}$ of the bunch train for different bunch lengths with elastic reflection of the electrons.

for the intermediate or long bunches. Correspondingly, also the central density is highest for intermediate bunch lengths, and not for the shortest (not shown, but see Ref. [2]). This indicates that electrons, once generated, can be more easily trapped by the electric potential of the longer bunches. To reveal the effect of the quadrupolar field component on the electron buildup, Fig. 4 compares simulation results for a pure dipole and for the PS combined function magnet. The evolution of the total electron line density is almost identical in the two cases. Also the local electron densities are only slightly different (not shown). Furthermore, the buildup has been simulated at different currents, to identify a possible intensity threshold. Figure 5 shows the evolution of the average cloud density for different bunch intensities, varying in the

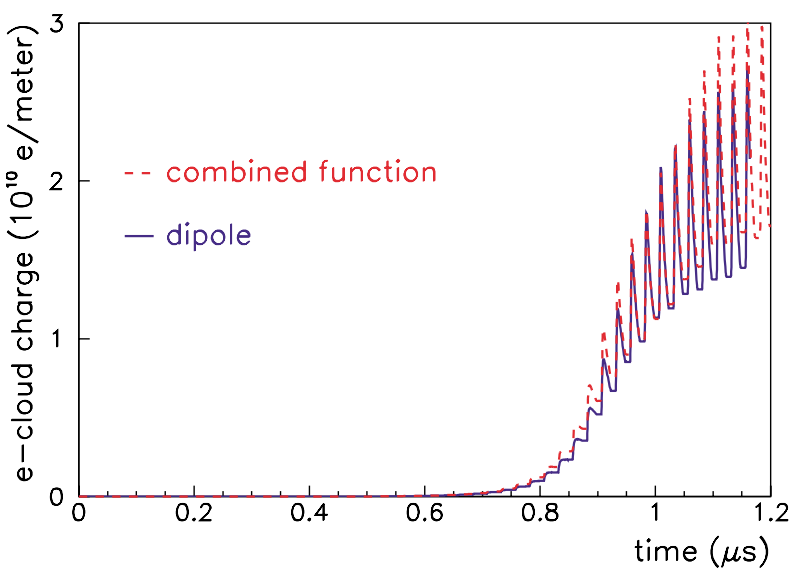

FIG. 4. (Color) Electron cloud buildup in a PS main magnet during the first $1.2 \mu \mathrm{s}$ of the bunch train with and without the field-gradient component. An rms bunch length of $2.5 \mathrm{~ns}$ has been considered with elastic reflection of the electrons. Both simulations were performed using a Runge-Kutta integration of the electron motion and included cyclotron oscillations in the magnetic field. 


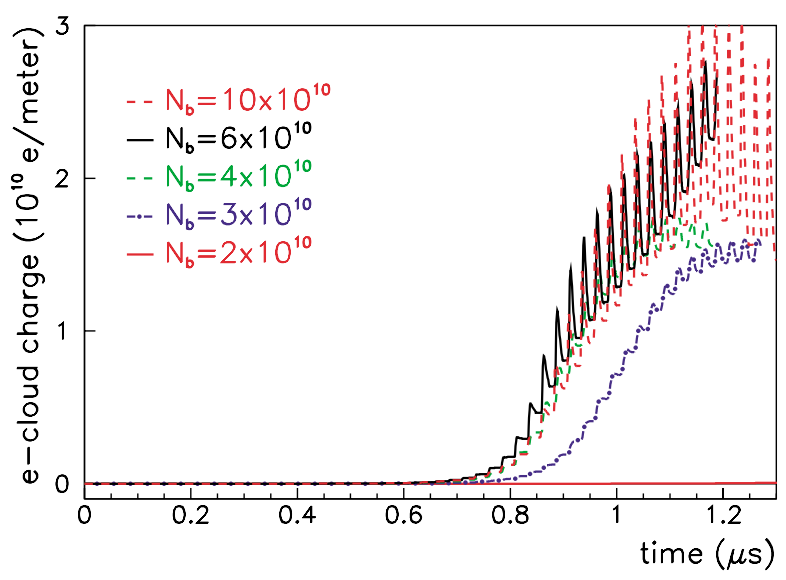

FIG. 5. (Color) Electron cloud buildup in a PS combined function magnet during the first $1.3 \mu \mathrm{s}$ of the bunch train for different bunch intensities. The electron cloud reaches saturation after the passage of about 50 bunches.

range $2-10 \times 10^{10} \mathrm{p} / \mathrm{b}$, and considering $\sigma_{z} / c=2.5 \mathrm{~ns}$. While the cloud rise time does not appear to be much affected by this parameter (after the passage of about 50 bunches, corresponding to $1.2 \mu \mathrm{s}$, the cloud has saturated for all cases with bunch intensities above $3 \times$ $10^{10} \mathrm{p} / \mathrm{b}$ ), the saturation level does not tend to grow significantly with increasing bunch current. The average saturated volume density amounts to $\left\langle\rho_{e, \text { sat }}\right\rangle=$ $\lambda_{e \text {, sat }} /\left(\pi h_{x} h_{y}\right)$, where $\lambda_{e \text {, sat }}$ denotes the line density in saturation and $h_{x}, h_{y}$ are the chamber half-apertures (see Table I). This average density is about $3 \times 10^{12} \mathrm{~m}^{-3}$ for bunch populations $N_{b}>4 \times 10^{10} \mathrm{p} / \mathrm{b}$.

It is worth emphasizing that the local electron density in the vicinity of the beam, relevant for the single-bunch instability discussed below, may strongly differ from the average density, since the electron buildup, which is due to a beam-induced multipacting process, occurs only in a restricted area of the vacuum chamber, with a typical

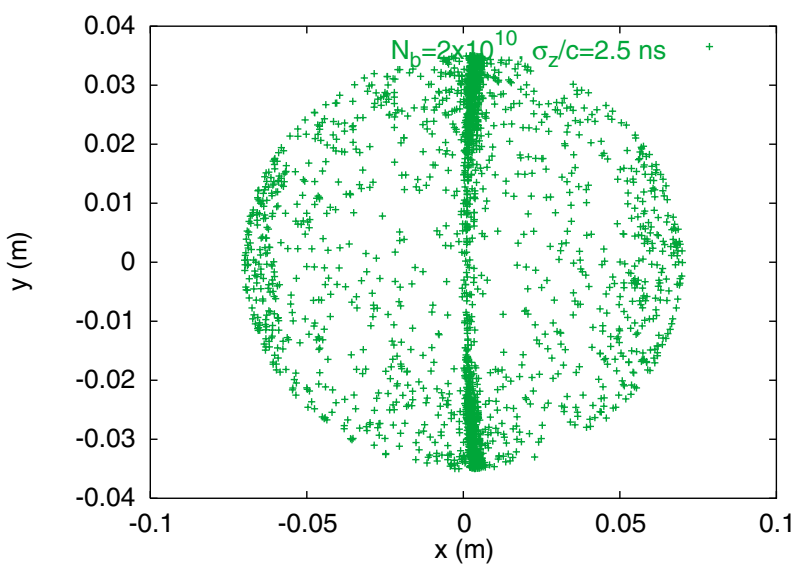

FIG. 6. (Color) Transverse electron distribution in a PS combined function magnet for $N_{b}=2 \times 10^{10} \mathrm{p} / \mathrm{b}$ and $\sigma_{z} / c=$ $2.5 \mathrm{~ns}$, after about $2 \mu \mathrm{s}$ (passage of 72 bunches).

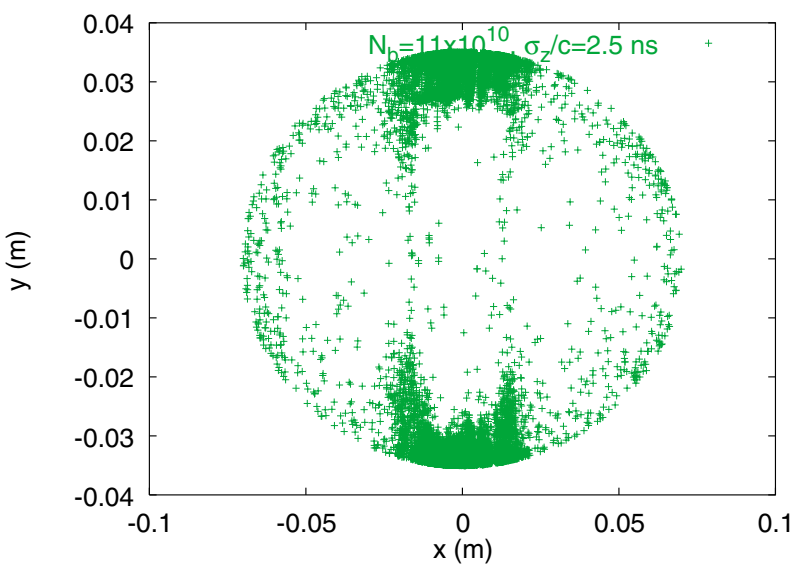

FIG. 7. (Color) Transverse electron distribution in a PS combined function magnet for $N_{b}=11 \times 10^{10} \mathrm{p} / \mathrm{b}$ and $\sigma_{z} / c=$ $2.5 \mathrm{~ns}$, after about $1 \mu \mathrm{s}$ (passage of 40 bunches).

form of one or two vertical "stripes" due to confinement in the strong dipole field. Simulated electron distributions for the PS combined function magnet and two different beam intensities are shown in Figs. 6 and 7. The generation of the primary electrons via gas ionization inside the beam may further add to the inhomogeneous distribution of electrons. Because of the stripelike electron distribution, the central densities can reach values up to 10 times larger than the average transverse densities. The nonuniformity of the electron distribution has been taken into account in the instability simulations presented in the next section.

\section{ELECTRON CLOUD INDUCED SINGLE- BUNCH INSTABILITY}

The electron cloud driven single-bunch instability and the associated wake fields for the PS have been studied using the HEADTAIL code. As input for these simulations we have assumed an electron cloud density of about $3 \times$ $10^{12} \mathrm{~m}^{-3}$, consistent with the density obtained by the simulations described above. The electron distribution was taken to be equal to a single stripe with a width of $4 \sigma_{x}$ and centered at the bunch axis. Note that the dependence of the simulated wake fields on the structure of the stripes (one or two), their distance, and width were explored in Ref. [14]. All the simulations reported here ignore the difference in the electron response between a combined function magnet and a field-free region, but model the entire ring as being covered by the combined function magnets. In reality, these magnets occupy about $70 \%$ of the PS circumference.

Instability simulations have been performed for a PS combined function magnet chamber. The aim of these simulations was to characterize the parameter regime for which an electron cloud instability could be excited in the PS. Scans with different bunch intensities, chromaticities, and bunch lengths were made in order to reveal the 
dependence of the instability on each of these parameters. If not mentioned otherwise, in these studies the rms bunch length has been set to $2.5 \mathrm{~ns}(0.75 \mathrm{~m})$, i.e., the value for which an instability was observed and monitored [3]. The chromaticity was assumed to be corrected in both planes.

We first evaluate the expected angular oscillation frequencies of electrons close to the bunch transverse center, $\omega_{x, y}$, and, from this, the number of oscillations $n_{x, y}$ that they perform during one bunch passage (over an effective bunch length $4 \sigma_{z}$ ). For a field-free region, these values can be computed as [15]

$$
\begin{gathered}
\omega_{e x(y)}=\sqrt{\frac{N_{b} r_{e} c^{2}}{2 \sigma_{x(y)} \sigma_{z}\left(\sigma_{x}+\sigma_{y}\right)}}, \\
n_{x(y)}=\frac{1}{\pi} \sqrt{\frac{2 N_{b} \sigma_{z} r_{e}}{\sigma_{x(y)}\left(\sigma_{x}+\sigma_{y}\right)}},
\end{gathered}
$$

where $r_{e}$ is the classical electron radius, $c$ the speed of light, and $\sigma_{x, y, z}$ the rms beam size (transverse and longitudinal, respectively). Considering a PS bunch containing $4 \times 10^{10}$ protons, Eqs. (2) and (3) yield $\omega_{e x}=$ $2 \pi \times 195 \mathrm{MHz}, \omega_{e y}=2 \pi \times 225 \mathrm{MHz}, n_{x}=1.95$, and $n_{y}=2.26$.

In a combined function magnet for which the vertical magnetic field component of the field is much stronger than the horizontal one, we may assume that in the vertical direction the electron oscillation frequency is given by Eq. (2). However, horizontally, the oscillation in the beam potential is suppressed by the strong vertical magnetic field. The latter gives rise to cyclotron motion around the almost vertical lines, which also is responsible for the stripelike regions of electron multiplication in Figs. 6 and 7. Figure 8 displays horizontal and vertical wake functions computed by considering a longitudinally

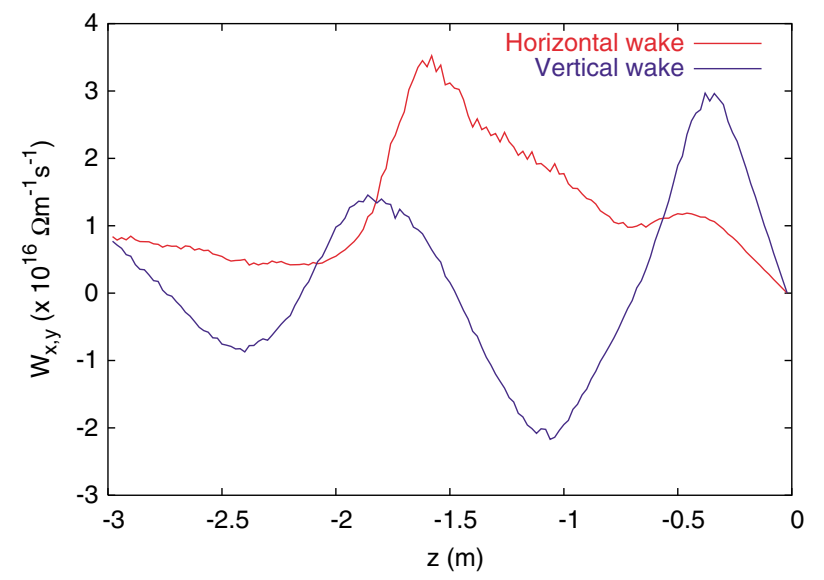

FIG. 8. (Color) Electron cloud horizontal and vertical wake functions for a longitudinally uniform bunch in a combined function magnet (nominal PS parameters, magnetic field gradient of $5.2 \mathrm{~T} / \mathrm{m})$. uniform beam distribution of constant line density $N_{b} /\left(4 \sigma_{z}\right)$, displacing the bunch head (longitudinally located at $z=0$ ) and calculating the average transverse force exerted on subsequent longitudinal bunch slices (the bunch center is at $-2 \sigma_{z}$, i.e., $-1.5 \mathrm{~m}$ in this example). We observe that, contrary to the case of a pure dipole field [16], the combined function magnet causes a significant horizontal wake whose frequency is much lower than that of the vertical one. The maximum wake amplitude strongly depends on the magnetic field gradient, as illustrated in Fig. 9, where the wakes for gradient values 10 times higher and lower than the nominal one are plotted.

Instead of considering a uniform bunch profile, we can also compute the wake field for a Gaussian bunch, again displacing the head and calculating the average force on subsequent longitudinal slices. The wake response for a Gaussian bunch profile so obtained is shown in Fig. 10, where we have assumed the PS beam parameters. Note that the computed wake depends on the longitudinal slice which is displaced, since both the electron density and the electron oscillation frequency vary along the bunch [14].
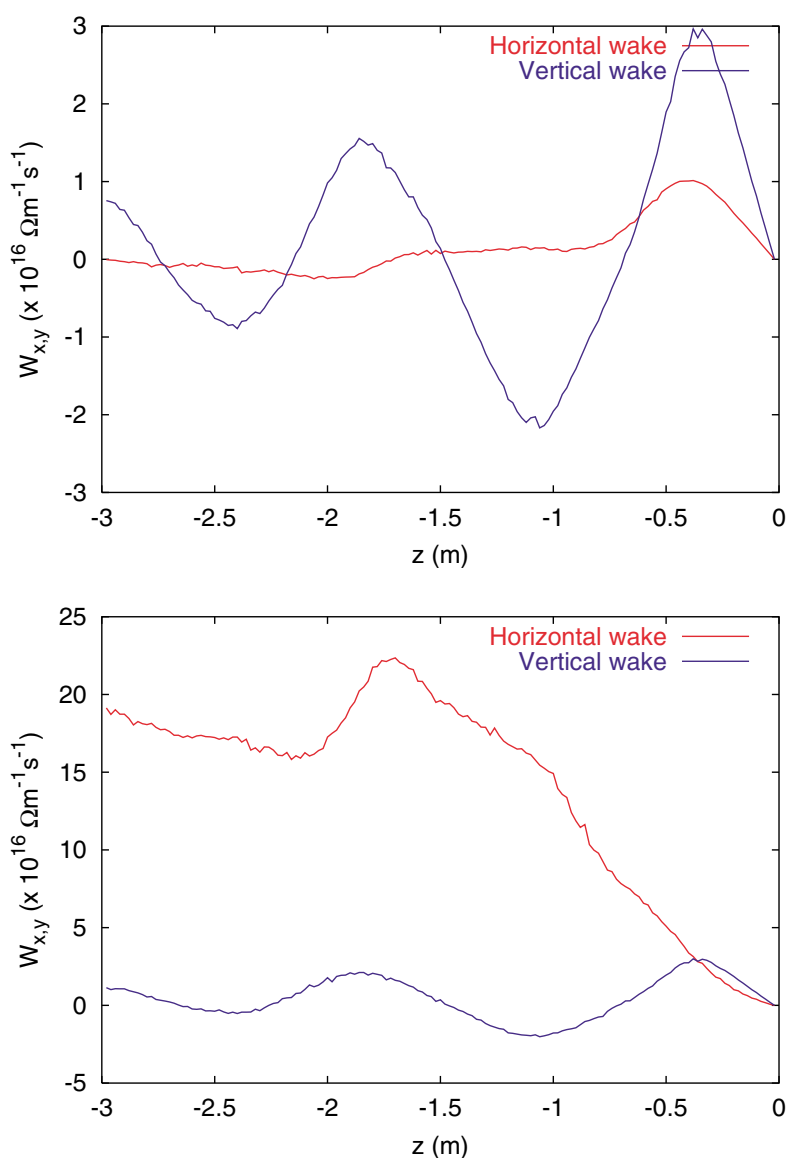

FIG. 9. (Color) Electron cloud horizontal and vertical wake functions for a longitudinally uniform bunch in a combined function magnet with magnetic field gradients of $0.52 \mathrm{~T} / \mathrm{m}$ (upper panel) and $52 \mathrm{~T} / \mathrm{m}$ (lower panel). 


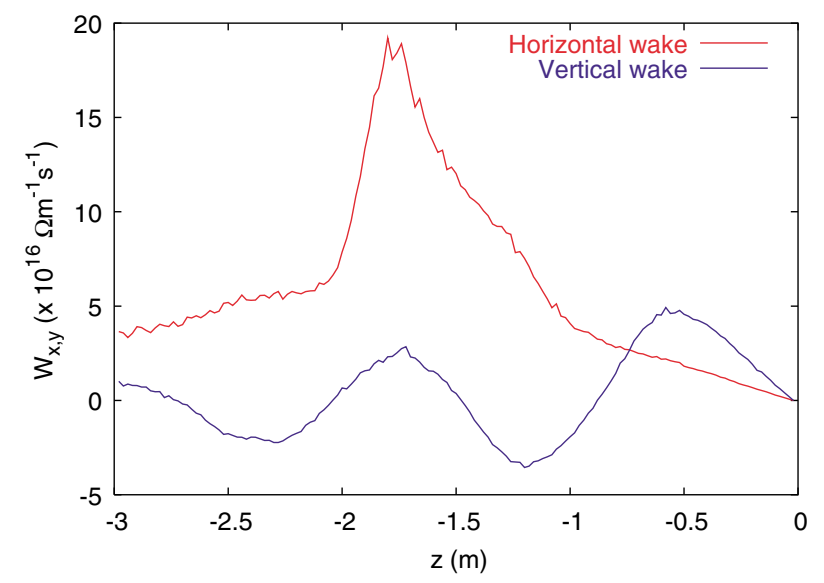

FIG. 10. (Color) Electron cloud horizontal and vertical wake functions for a Gaussian bunch of $2.5 \mathrm{~ns}$ rms length $(0.75 \mathrm{~m})$ in a combined function magnet (nominal magnetic field gradient of $5.2 \mathrm{~T} / \mathrm{m})$.

At this stage, it is useful to point out that the studies of wake field and instability were conducted considering the electron motion as frozen around the slightly bent field lines. This is because, due to the very strong dipole field component, for an exact calculation of the electron motion the electrons would narrowly spiral around the field lines, such that the computation would require very short time steps and excessively long simulation times. Unlike for a pure dipole, the approximation that the electron motion is frozen around the field lines may not be fully adequate for the combined function magnet, since this approximation neglects effects like the magnetic mirroring due to the spatial field inhomogeneity $[17,18]$ (see also Ref. [19] for a recent analysis of magnetic trapping by different magnetic fields in electron cloud buildup simulations). The underlying assumption was that these effects do not much influence the electron dynamics at the moment of the bunch passage, because of both the strong beam electric field and the large dipolar component of the magnetic field.

Figure 11 shows the horizontal (upper panel) and vertical (lower panel) emittance growth due to the electron cloud over 2000 turns for different bunch intensities. The rise time of the vertical instability is of the order of a few milliseconds, spanning between $2 \mathrm{~ms}$ for the highest intensity $\left(N_{b}=10 \times 10^{10} \mathrm{p} / \mathrm{b}\right)$ and 3-4 ms for a bunch population of $N_{b}=4 \times 10^{10} \mathrm{p} / \mathrm{b}$. Note that, in this paper, the instability rise time is defined in terms of emittance growth and not in terms of beam-size increase, hence such a rise time will be 2 times smaller than the classical one. The instability appears more pronounced in the vertical plane. Horizontally, the unstable motion starts at a later time. Nevertheless, because of the tilted field lines in a combined function magnet the beam can grow unstable also in the horizontal plane, which is different from a pure dipole field. A threshold for the instability onset of about $N_{b}=3 \times 10^{10} \mathrm{p} / \mathrm{b}$ is clearly visible in the
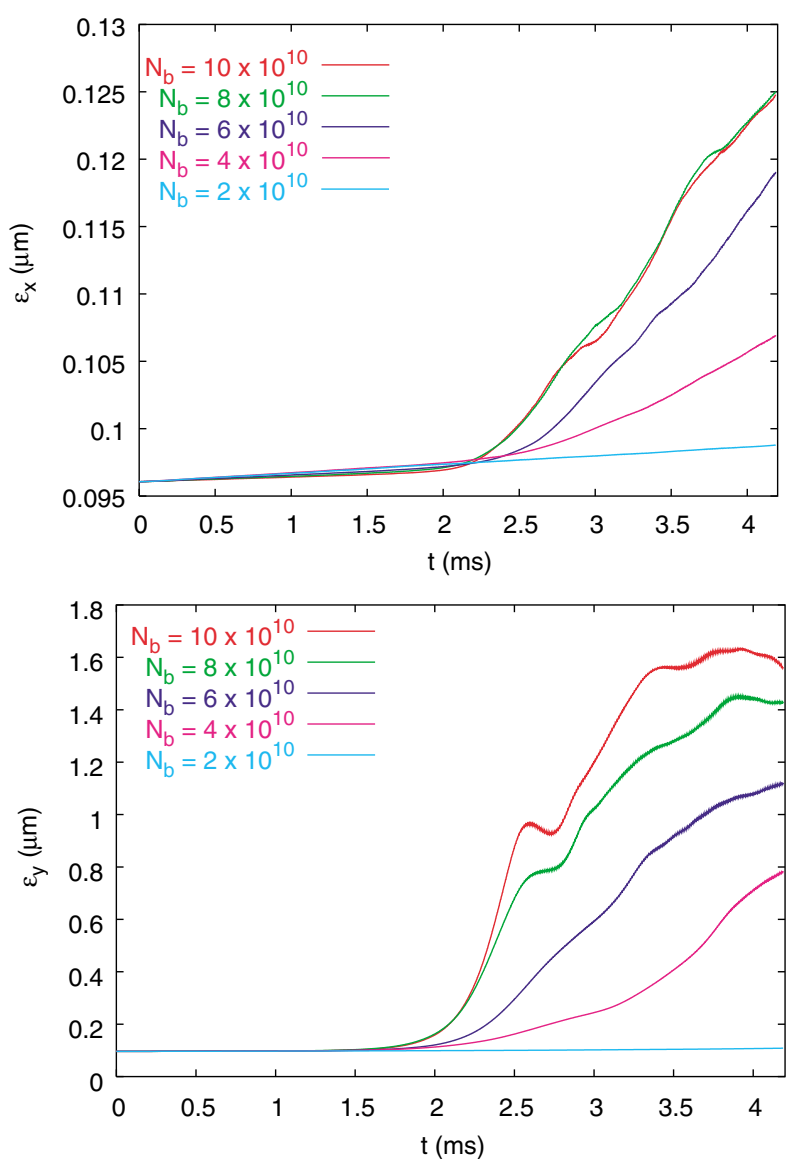

FIG. 11. (Color) Horizontal (upper panel) and vertical (lower panel) emittance growth over 2000 turns for different bunch intensities and zero chromaticity (different vertical scales are used in the two pictures).

vertical plane. Figure 12 illustrates the simulated emittance growth for a bunch with $N_{b}=4 \times 10^{10} \mathrm{p} / \mathrm{b}$ and for different values of (positive) chromaticity. These pictures suggest that a positive chromaticity larger than 0.3 can efficiently suppress the degrading effect of the electron cloud. In both planes the instability growth time decreases for increasing values of $\xi$ up to 0.25 , and the bunch becomes stable for higher values. Similarly, at higher current $\left(N_{b}=8 \times 10^{10} \mathrm{p} / \mathrm{b}\right)$, increasing the chromaticity to $\xi \approx 0.5$ can still reduce the instability growth rate or render the bunch stable. An example is shown in Fig. 13.

Finally, the effect of the bunch length has been studied by using a rematching procedure that models the PS situation. The bunch is longitudinally rematched to the bucket by maintaining a constant longitudinal emittance, and, therefore, multiplying its synchrotron tune by the square of the ratio $\sigma_{z}^{\text {old }} / \sigma_{z}^{\text {new }}$. Following this procedure, we find that for low current, the bunch length $\sigma_{z}^{\text {old }}=$ $2.5 \mathrm{~ns}$ is at the limit of the region where an instability occurs (see Fig. 14); shorter bunches are stable. For higher current $\left(N_{b}=8 \times 10^{10} \mathrm{p} / \mathrm{b}\right)$, the threshold decreases, as shown in Fig. 15. 

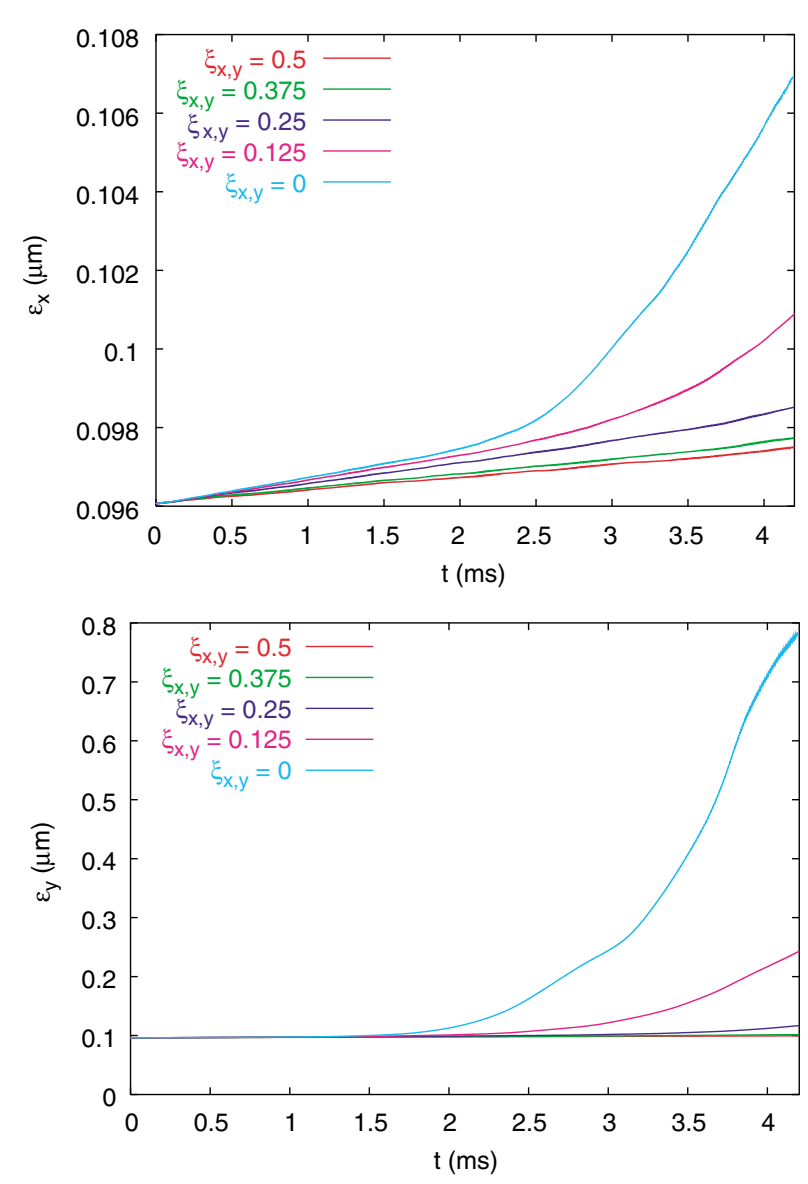

FIG. 12. (Color) Horizontal (upper panel) and vertical (lower panel) emittance growth over 2000 turns for different chromaticities and $N_{b}=4 \times 10^{10} \mathrm{p} / \mathrm{b}$. This bunch intensity is slightly above the estimated instability threshold for zero chromaticity (different vertical scales are used in the two pictures).

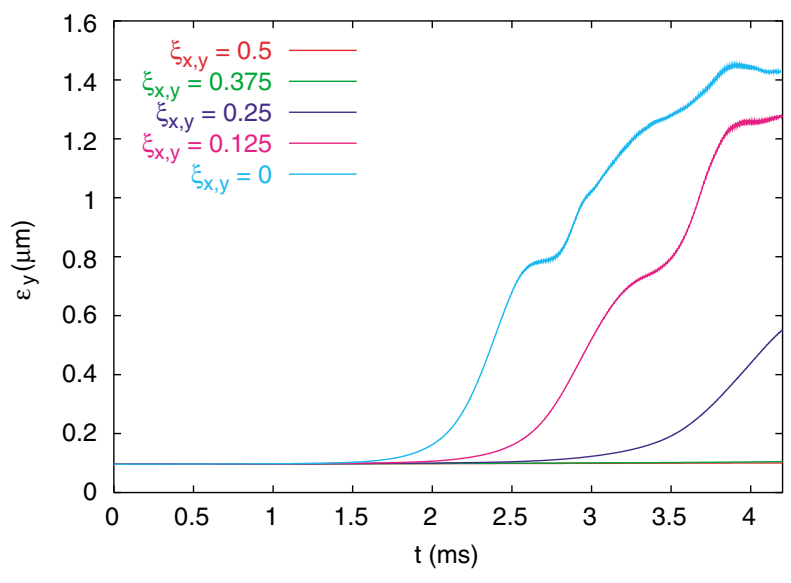

FIG. 13. (Color) Vertical emittance growth over 2000 turns for different chromaticities and $N_{b}=8 \times 10^{10} \mathrm{p} / \mathrm{b}$. This bunch intensity is far inside the instability region.
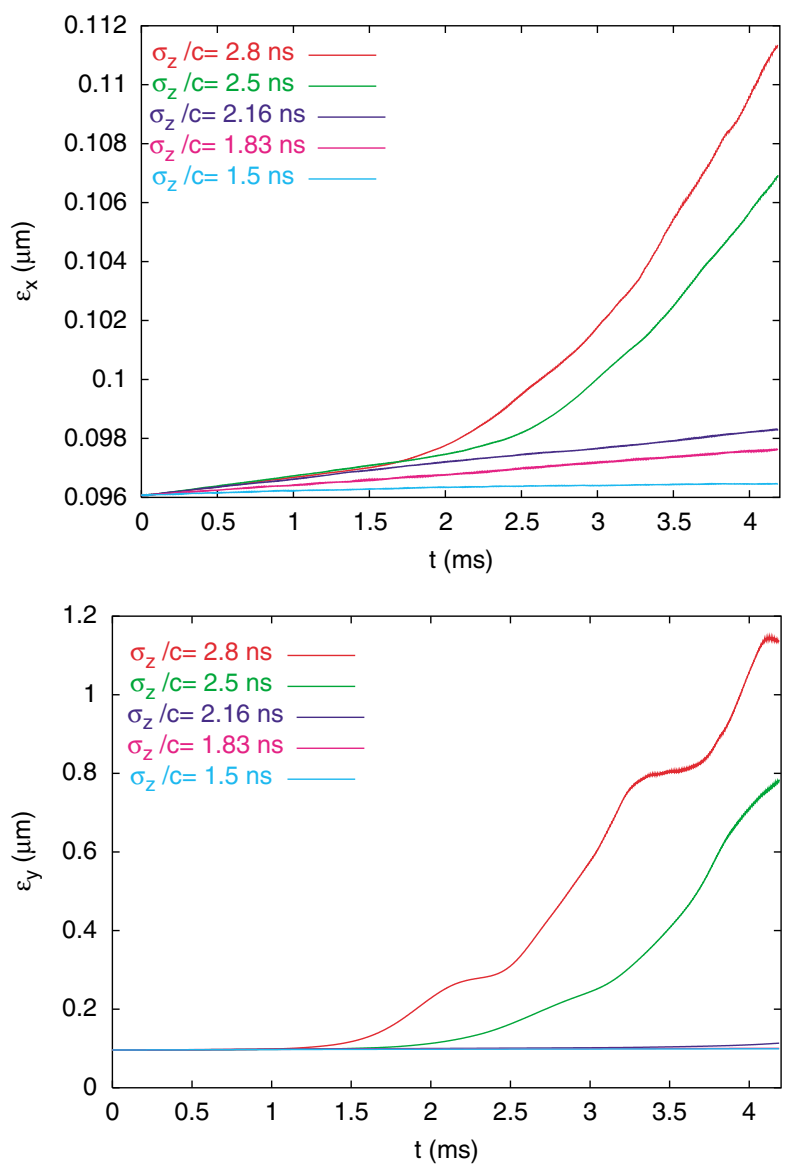

FIG. 14. (Color) Horizontal (upper panel) and vertical (lower panel) emittance growth over 2000 turns for different bunch lengths and $N_{b}=4 \times 10^{10} \mathrm{p} / \mathrm{b}$. For each case, the bunch has been rematched by keeping a constant longitudinal emittance and adjusting the synchrotron tune (different vertical scales are used in the two pictures).

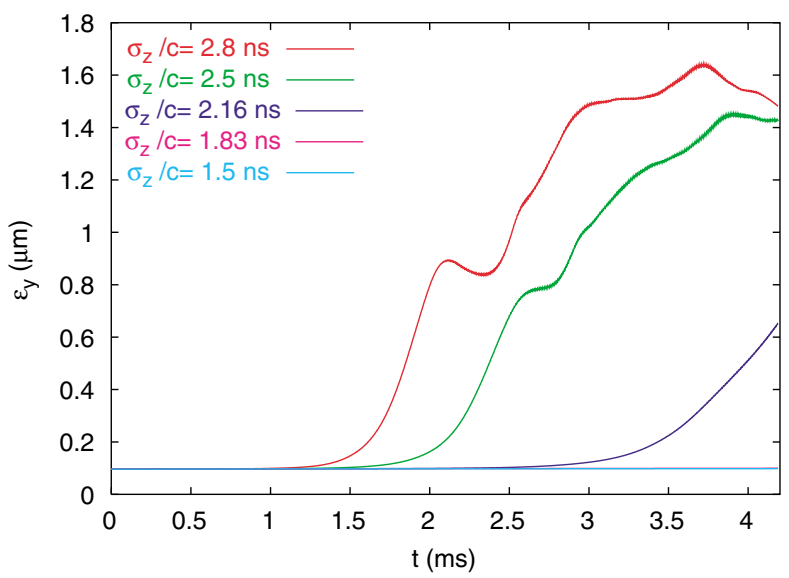

FIG. 15. (Color) Vertical emittance growth over 2000 turns for different bunch lengths and $N_{b}=8 \times 10^{10} \mathrm{p} / \mathrm{b}$. The bunch has been rematched for each case by keeping the longitudinal emittance constant and changing the synchrotron tune. 


\section{COMPARISON WITH OBSERVATIONS}

The observed horizontal instability rise time of a few milliseconds is compatible with the simulated growth rates. Although the simulation for a single-bunch passage through the electron cloud in a combined function magnet does show a strong horizontal wake, the simulation of the emittance evolution over many turns predicts a faster growth in the vertical direction. Indeed, for all the cases that we have examined in this study, the rise time of the instability is shorter in the vertical plane, and the related vertical emittance increase over 2000 turns is also always significantly larger. However, by contrast, in the experiment only a horizontal instability was observed [3]. Indeed, by closely looking at the emittance-growth curves (Fig. 12), one may observe that for a chromaticity value of about $\xi_{x} \approx \xi_{y} \approx 0.2$, the growth rate is roughly the same in both planes. In fact, this is the situation best fitting the actual experimental conditions, where the chromaticities are about $0.1-0.2$ [3]. Therefore, this seems to indicate that the agreement between numerical simulations and experimental results is within reach. In this respect, a sensitivity study, i.e., a study of the robustness of the simulation results against small variations of the relevant physical parameters, might be extremely useful.

In the simulation, higher positive chromaticity suppresses the instability, whereas experimentally no significant change of the instability behavior was observed, when the horizontal chromaticity was varied by several tenths of units [3]. It is worthwhile stressing that the PS observations are not in agreement with the predictions of numerical simulations.

In future simulation studies aimed at resolving this discrepancy, we plan to consider a more accurate model of the electron motion during the bunch passage and finer details of the electron cloud distribution. However, it does not seem likely that these refinements will alter the qualitative conclusions. We rather suspect that either some of the beam or storage-ring parameters differ from the assumed values (for example, the chromaticities), or that an important physics ingredient is still missing in our simulation for the PS machine. Possible candidates are the nonlinear components of the magnetic field generated by the combined function dipole.

\section{CONCLUSIONS}

Simulations have been carried out with the ECLOUD and HEADTAIL codes to interpret the electron cloud observations at the CERN PS. The results show that a train of bunches with the LHC nominal spacing is expected to produce an electron cloud in the PS chamber for rms bunch lengths in the range $1-3 \mathrm{~ns}$. The equilibrium value reached by the cloud density at saturation is then a function of the bunch intensity and length, showing saturation values around $3 \times 10^{12} \mathrm{~m}^{-3}$ for bunch populations in the range $1-10 \times 10^{10}$ protons and $\sigma_{z} / c=2.5 \mathrm{~ns}$. Such an electron cloud, supposed to be uniformly distributed in the longitudinal direction all along the ring, is able to render the single bunch unstable on a time scale of a few milliseconds. The simulation shows that the instability threshold lies around $3 \times 10^{10} \mathrm{p} / \mathrm{b}$. For lower intensities no significant emittance growth is expected; for higher intensities the vertical emittance may increase by a factor as large as 20 with a rise time of a few milliseconds that becomes shorter as the current is increased. In the simulation, increasing the chromaticity seems to be an efficient cure against this kind of instability. Positive values of $\xi$ above 0.5 can completely suppress the simulated instability both for moderate and higher currents. Furthermore, it was found that shorter bunches and higher synchrotron tunes have a stabilizing effect on the electron cloud. A bunch with $1 \mathrm{~ns}$ rms length is expected to remain stable even for a population of $10 \times 10^{10}$ protons. Unfortunately, this case could not be studied in practice for a stored beam in the PS machine, since the short $1 \mathrm{~ns}$ long rms bunch length can be generated only by a nonadiabatic process just prior to extracting the LHC beam from the PS towards the Super Proton Synchrotron [3].

Simulations of the electron cloud response to a head displacement indicate that a significant horizontal wake field may exist in a combined function magnet, in contrast to the case of a pure dipole field, where the horizontal wake is close to zero. While multiturn instability simulations carried out for a combined function magnet suggest that the beam blowup occurs mainly in the vertical plane, a horizontal emittance growth is also predicted, albeit on a slightly longer time scale. Thus the numerical simulations indicate that the large horizontal wake field alone cannot fully explain the observation of a strong instability in the horizontal plane only [3]. Work is presently underway and more measurements have been planned at the PS ring to achieve a better comprehension of this phenomenon.

\section{ACKNOWLEDGMENTS}

The authors would like to thank R. Cappi, K. Ohmi, M. Martini, F. Ruggiero, and D. Schulte for helpful discussions and information.

[1] G. Rumolo, F. Ruggiero, and F. Zimmermann, Phys. Rev. ST Accel. Beams 4, 012801 (2001).

[2] R. Cappi, M. Giovannozzi, E. Métral, G. Métral, and F. Zimmermann, in Proceedings of the 2001 Particle Accelerator Conference, Chicago, IL, edited by P.W. Lucas and S. Webber (IEEE, Piscataway, NJ, 2001), p. 682 . 
[3] R. Cappi, M. Giovannozzi, E. Métral, G. Métral, G. Rumolo, and F. Zimmermann, Phys. Rev. ST Accel. Beams 5, 094401 (2002).

[4] "Electron Cloud in the LHC" web page, http:// wwwslap.cern.ch/collective/electron-cloud/.

[5] G. Rumolo and F. Zimmermann, CERN Report No. SL 2001-067 (AP), 2001 (unpublished).

[6] F. Zimmermann and G. Rumolo, in Proceedings of the Mini-Workshop on Electron-Cloud Simulations for Proton and Positron Beams, CERN, Geneva, 2002, edited by G. Rumolo and F. Zimmermann (unpublished).

[7] G. Rumolo and F. Zimmermann, in Proceedings of the Mini-Workshop on Electron-Cloud Simulations for Proton and Positron Beams, CERN, Geneva, 2002 (Ref. [6]).

[8] T. Risselada, CERN PS (PA) Note No. 92-04, 1992 (unpublished).

[9] D. Cornuet and Z. Sharifullin, CERN AT (MA) Note No. 92-23, 1992 (unpublished).

[10] D. Manglunki and M. Martini, CERN PS Report No. 97018 (CA), 1997 (unpublished).

[11] V. Baglin, J. Bojko, O. Grobner, B. Henrist, N. Hilleret, C. Scheuerlein, and M. Taborelli, in Proceedings of the 2002 European Particle Accelerator Conference, Paris, France, edited by J. Poole and C. Petit-Jean-Genaz (European Physical Society and CERN, Geneva, 2002), p. 217.
[12] V. Baglin, I. Collins, B. Henrist, N. Hilleret, and G. Vorlaufer, LHC Project Report No. 472, 2001 (unpublished).

[13] F. Zimmermann, in Proceedings of the Mini-Workshop on Electron-Cloud Simulations for Proton and Positron Beams, CERN, Geneva, 2002 (Ref. [6]).

[14] G. Rumolo and F. Zimmermann, in Proceedings of the 2002 European Particle Accelerator Conference, Paris France (Ref. [11]), p. 1556.

[15] G. Rumolo and F. Zimmermann, in Proceedings of LHC Workshop - Chamonix XI, Chamonix, 2001, edited by J. Poole (unpublished).

[16] L. Wang, G. Rumolo, K. Ohmi, and F. Zimmermann, in Proceedings of the Second Asian Particle Accelerator Conference, Beijing, China, 2001, edited by C. Zahng et al. (IHEP, Beijing, 2002), p. 442.

[17] H. Alfvén and C.G. Fälthammar, Cosmical Electrodynamics: Fundamental Principles (Clarendon Press, Oxford, 1963).

[18] J. D. Jackson, Classical Electrodynamics (John Wiley \& Sons, New York, 1998).

[19] L. F. Wang, H. Fukuma, K. Ohmi, S. Kurokawa, K. Oide, and F. Zimmermann, in Proceedings of the Mini-Workshop on Electron-Cloud Simulations for Proton and Positron Beams, CERN, Geneva, 2002 (Ref. [6]). 